\title{
KASNER-LIKE REGIONS NEAR CRUSHING SINGULARITIES
}

\author{
JOHN LOTT
}

\begin{abstract}
We consider vacuum spacetimes with a crushing singularity. Under some scale-invariant curvature bounds, we relate the existence of Kasner-like regions to the asymptotics of spatial volume densities.
\end{abstract}

\section{INTRODUCTION}

This paper is about the initial geometry of an expanding vacuum spacetime. In Subsection 1.1 of this introduction we give some background about the problems addressed. In Subsection 1.2 we describe some of the techniques used. In Subsection 1.3 we give broad descriptions of the results. A reader who is interested in the main results can skip to Subsection 1.4.

1.1. Scenario. We are concerned with expanding spacetimes that satisfy the vacuum Einstein equations. The word "expanding" refers to spatial slices and its meaning will be clarified. One can study the asymptotic geometry of such a spacetime in either the future or past directions. The problems that one studies in the two directions are rather different.

In the future direction, one can ask, for example, whether the geometry becomes asymptotically homogeneous; see [12, 19] and references therein. In the other direction, Hawking's singularity theorem says that under fairly general assumptions, an expanding spacetime has a past singularity, in the sense of an incomplete timelike geodesic [14, Theorem 4 on p. 272]. One basic question, relevant for the strong cosmic censorship question, is whether the curvature generically blows up; see [15] and references therein. We are concerned with a somewhat different question, namely the nature of the geometry as one approaches the singularity.

As a remark about the physical relevance of considering vacuum spacetimes, under some assumptions there are heuristic arguments to say that the matter content is irrelevant for the past asymptotics [6, Chapter 4]. Hence to simplify things, we only consider vacuum spacetimes with vanishing cosmological constant.

One special but relevant class of vacuum spacetimes comes from the Milne spacetimes. In four dimensions, a Milne spacetime is a flat spacetime that is a quotient of a forward lightcone in Minkowski space. Equivalently, it is the Lorentzian cone over a three dimensional Riemannian manifold of constant sectional curvature -1 . We define an $(n+1)$ dimensional spacetime to be of Milne type if it is a Lorentzian cone over a Riemannian

Date: November 30, 2020.

Research partially supported by NSF grant DMS- 1810700 . 
$n$-manifold whose Ricci tensor is $-(n-1)$ times the Riemannian metric; the spacetime is then a vacuum spacetime.

Another special but relevant class of vacuum spacetimes consists of the Kasner spacetimes, which exist in any dimension; see Example 4.5. They are usually not flat but they are self-similar, in the sense that they admit a timelike homothetic Killing vector field. More generally, one has the spatially homogeneous Bianchi spacetimes. Among these, the so-called Mixmaster spacetimes [22, Chapter 30.7] have a left-SL(2, $\mathbb{R})$ spatial invariance (Bianchi VIII) or a left-SU(2) spatial invariance (Bianchi IX).

The strongest results about geometric asymptotics, in the future or past, assume some continuous symmetries. Going beyond this, one has the BKL conjectures for the asymptotics of a generic vacuum spacetime with an initial singularity, as one approaches the singularity [5, 6]. The conjectures are loosely formulated but contain the following points:

(1) The evolution at different spatial points asymptotically decouples.

(2) For a given spatial point, the asymptotic evolution is governed by the ODE of a homogeneous vacuum spacetime of Bianchi type VIII or IX.

Generic Bianchi VIII and Bianchi IX vacuum spacetimes have been extensively studied, with rigorous results in [4, 7, 9, 17, 24]. Approaching the singularity, they have time regions of Kasner-like geometry, with jumps from one Kasner-like geometry to another. The jumps are along Bianchi II trajectories and occur chaotically, when viewed on the right time scale.

There does not seem to be strong evidence either way regarding the BKL conjectures, although numerics indicate some positive aspects. In this paper we focus on the Kasnerlike regions. Rather than considering generic vacuum spacetimes, we look for conditions that ensure the existence of Kasner-like regions, and conditions that rule them out.

1.2. Techniques. Our approach to analyzing the future or past behavior of vacuum spacetimes consists of three features.

- A way to rescale vacuum spacetimes, that allows one to consider blowdown or blowup limits.

- A monotonicity result.

- A way to take a convergent subsequence of a sequence of vacuum spacetimes.

In combination, one can use these features to prove results about geometric asymptotics of a spacetime by contradiction. One identifies a class of putative target spacetimes and assumes that a sequence of (blowdown or blowup) rescalings of the given vacuum spacetime do not approach the target class. One takes a convergent subsequence of the rescalings and uses the monotonicity result to show that the limit does in fact lie in the target class, thereby obtaining a contradiction. This general approach is used to study many geometric flows, such as the Ricci flow. For the future behavior of vacuum spacetimes, it was pioneered by Anderson [1].

In order to make progress, we make a standard assumption that the vacuum spacetime in question has a foliation by constant mean curvature (CMC) spatial hypersurfaces. We assume that the spacetime is expanding, meaning that the spatial slices have negative mean curvatures that increase toward the future. Examples in which CMC foliations are known 
to occur come from crushing singularities, meaning that in the past there is a sequence of compact Cauchy surfaces whose mean curvatures approach $-\infty$ uniformly [10, 21]. If there is a crushing singularity then there is a CMC foliation in the past by compact hypersurfaces, whose mean curvatures $H$ approach $-\infty$ [13].

If the spacetime has dimension $n+1$, define the Hubble time by $t=-\frac{n}{H}$. Let $X$ denote the spatial manifold, with Riemannian metric $h(t)$. Given a parameter $s>0$, there is a natural way to rescale the CMC vacuum spacetime to produce another one; see (2.13).

If $X$ is compact then Fischer and Moncrief proved the remarkable monotonicity statement that $t^{-n} \operatorname{vol}(X, h(t))$ is nonincreasing in $t$ [12]; a closely related monotonic quantity was considered by Anderson [1]. More precisely,

$$
\frac{d}{d t}\left(t^{-n} \operatorname{vol}(X, h(t))\right)=-t^{1-n} \int_{X} L\left|K^{0}\right|^{2} \operatorname{dvol}_{h}
$$

where $L$ is the lapse function and $K^{0}$ is the traceless second fundamental form of the hypersurface. One shows that $t^{-n} \operatorname{vol}(X, h(t))$ is constant in $t$ if and only if the vacuum spacetime is a Milne spacetime. Based on this, Fischer and Moncrief suggested that most of a spacetime, in the sense of volume, approaches a Milne spacetime in the future. Actually, this is true relative to a limiting spatial volume density $\operatorname{dvol}_{\infty}=\lim _{t \rightarrow \infty} t^{-n} \operatorname{dvol}_{h(t)}$. Related to this, the future stability of Milne spacetimes with compact spatial slices was shown by Andersson and Moncrief in [2, 3.

It is also possible that $\mathrm{dvol}_{\infty}$ vanishes, in which case the future asymptotics have different models and were studied in [19].

Because $t^{-n} \operatorname{vol}(X, h(t))$ is nonincreasing in $t$, it is bounded above for large $t$ and so it is suitable for studying future behavior. On the other hand, to study past behavior, one wants an expression that is monotonic in the other direction. In [20] it was shown that $t^{-1} \operatorname{vol}(X, h(t))$ is nondecreasing in $t$ provided that the spatial scalar curvature $R$ is nonpositive. More precisely,

$$
\frac{d}{d t}\left(t^{-1} \operatorname{vol}(X, h(t))\right)=-\frac{1}{n} \int_{X} L R \operatorname{dvol}_{h}
$$

If $X$ is a compact 3-manifold with contractible universal cover, for example if it is diffeomorphic to a torus, and if $R \leq 0$ then $t^{-1} \operatorname{vol}(X, h(t))$ is constant in $t$ if and only if the vacuum spacetime is a Kasner spacetime. Thus the expression $t^{-1} \operatorname{vol}(X, h(t))$ plays a parallel role to the Fischer-Moncrief expression $t^{-n} \operatorname{vol}(X, h(t))$, in terms of characterizing model geometries.

One can refine the monotonicity results to statements that are pointwise with respect to the spatial directions [19, (2.16)],[20, Corollary 5]. That is, one has monotonicity along any timelike curve that intersects the spatial hypersurfaces orthogonally. In this case, one does not have to assume spatial compactness.

The next issue is about taking a convergent subsequence of a sequence of spacetimes. In the static case of Riemannian manifolds, there is a well developed theory of (pre)compactness under uniform sectional curvature bounds, as explained in [23, Chapter 11]. An extension 
to vacuum CMC spacetimes was developed by Anderson, who applied it to future asymptotics [1]. In order to extract a blowdown limit, i.e. a subsequential limit of a sequence of rescaled spacetimes, the curvature assumption was the scale-invariant condition that the curvature is $O\left(t^{-2}\right)$ as $t \rightarrow \infty$. We review this material in Subsection 2.2. In our case, to extract a blowup limit, the curvature assumption becomes that the curvature is $O\left(t^{-2}\right)$ as $t \rightarrow 0$. Following Ricci flow terminology, we call this a type-I vacuum solution.

1.3. Objectives. The goal of this paper is to give information about the geometry of an expanding vacuum spacetime near an initial singularity, without any symmetry assumptions. We assume the existence of a CMC foliation. We also assume a type-I curvature bound. We find that the rate of decay of the spatial volume density leads to information about the local geometry. For example, if the spatial volume density decays at the fastest possible rate as one approaches the singularity, namely $t^{n}$, then we show that the local geometry is asymptotically of the Milne type.

Motivated by the BKL conjectures, we are also interested in characterizing Kasnertype regions. As an improvement to [20], rather than assuming nonpositive spatial scalar curvature, we just assume that the spatial scalar curvature is asymptotically nonpositive; this seems to often be the case. Under this assumption, we show that if the spatial volume density strictly decays at the slower rate $t^{1}$ then the local geometry is asymptotically Kasner-like as one approaches the singularity.

For the Mixmaster solutions that arise in the BKL conjectures, the decay of the spatial volume density is not strictly $t^{1}$. In fact, the decay is a bit faster, because of the Bianchi-II transitions between the Kasner-type regions. In order to cover this situation, we consider the case when the spatial volume density is $O(t)$ as $t \rightarrow 0$, but is not $O\left(t^{1+\beta}\right)$ for any $\beta>0$. (An example to have in mind is $\frac{t}{\log \frac{1}{t}}$.) In this case we show that in a technical sense, almost all of the time along a trajectory approaching the singularity is spent in Kasner-like geometries.

The overall theme is that under some reasonable a priori assumptions, a condition about an object of low regularity, the spatial volume density, leads to conclusions about the local geometry as one approaches the singularity.

1.4. Main results. We consider vacuum spacetimes that are diffeomorphic to $\left(0, t_{0}\right] \times X$, where $X$ is an $n$-dimensional manifold, possibly noncompact. After performing spatial diffeomorphisms, the spacetime metric takes the form $g=-L^{2} d t^{2}+h(t)$, where $L$ is the lapse function and $h(\cdot)$ is a family of Riemannian metrics on $X$.

We assume that each spatial slice has constant mean curvature, so we have a CMC foliation. As mentioned before, this is the case in the neighborhood of a crushing singularity. We are interested in the expanding case, so we assume that the mean curvature $H$ is monotonically increasing in $t$ and takes all values in $\left(-\infty, H_{0}\right)$ for some $H_{0}<0$. We can then use the Hubble time given by $t=-\frac{n}{H}$.

In terms of the time evolution, we can alternatively think of a vacuum spacetime with a spatial foliation as a flow, the Einstein flow. Let $|\mathrm{Rm}|_{T}$ be the pointwise curvature norm defined in (2.14). 
Definition 1.3. A type-I Einstein flow is a CMC Einstein flow for which there is some $C<\infty$ so that $|\operatorname{Rm}|_{T} \leq C t^{-2}$ for all $t \in\left(0, t_{0}\right]$.

Given an Einstein flow and a parameter $s>0$, there is a rescaled Einstein flow $\mathcal{E}_{s}$, defined in (2.13), with $s \rightarrow 0$ corresponding to a blowup limit as one approaches the singularity at time zero. The inequality in Definition 1.3 is scale-invariant.

Our first result gives a situation where one can rule out the existence of Kasner-like regions. It describes the $t \rightarrow 0$ asymptotics at a point $x \in X$ where the spatial volume density $\operatorname{dvol}_{h(t)}(x)$ has the scale invariant behavior $O\left(t^{n}\right)$. Since we localize around $x$, we use the notion of a pointed manifold, meaning a manifold with a specified basepoint. Let $\mathcal{M}$ denote the space of pointed Einstein flows that correspond to Lorentzian cones over pointed Riemannian Einstein $n$-manifolds with Einstein constant $-(n-1)$. In what follows, $p$ will range over $[1, \infty)$ and $\alpha$ will range over $(0,1)$. The relevant notions of pointed convergence are defined in Subsection 2.2.

Theorem 1.4. Suppose that $\mathcal{E}$ is a type-I CMC Einstein flow. Fix $x \in X$. Suppose that $\operatorname{dvol}_{h(t)}(x)$ is $O\left(t^{n}\right)$ as $t \rightarrow 0$. Then as $s \rightarrow 0$, the rescaled Einstein flows $\mathcal{E}_{s}$, pointed at $x$, approach $\mathcal{M}$ in the pointed weak $W^{2, p}$-topology and the pointed $C^{1, \alpha}$-topology.

Theorem 1.4 describes the type-I Einstein flows with the fastest possible volume shrinkage as $t \rightarrow 0$. To deal with some Einstein flows with a slower volume shrinkage, we introduce another class of special Einstein flows. Let $K$ denote the second fundamental form, let $R$ denote the spatial scalar curvature and let $\mathcal{K}$ be the collection of pointed expanding type-I CMC Einstein flows with $R=0, L=\frac{1}{n}$ and $|K|^{2}=H^{2}$, defined for $t \in(0, \infty)$. Examples of such Einstein flows are the $n$-dimensional Kasner flows (Example 4.5). If $X$ is a compact orientable 3-manifold, and there is an aspherical component in the prime decomposition of $X$ (for example, if $X$ is diffeomorphic to $T^{3}$; see [25, §6] for definitions), then an element of $\mathcal{K}$ is a Kasner flow in the sense of Example 4.5 [20, Proposition 9]. In particular, it is spatially flat. We do not know if this is true more generally, say if $X$ is a noncompact 3-manifold and the spatial slices are complete. So we will think of elements of $\mathcal{K}$ as Kasner-like Einstein flows.

Definition 1.5. The $C M C$ Einstein flow $\mathcal{E}$ has asymptotically nonpositive spatial scalar curvature if $\lim \sup _{t \rightarrow 0} \sup _{x \in X} t^{2} R(t, x) \leq 0$.

The next theorem gives a sufficient condition to ensure that as $t \rightarrow 0$, the geometry becomes increasingly Kasner-like.

Theorem 1.6. Let $\mathcal{E}$ be a type-I CMC Einstein flow with asymptotically nonpositive spatial scalar curvature. Suppose in addition that there is a nonnegative function $\widehat{R}:\left(0, t_{0}\right] \rightarrow \mathbb{R}$ with $\int_{0}^{t_{0}} t^{2} \widehat{R}(t) \frac{d t}{t}<\infty$ so that $R(t, x) \leq \widehat{R}(t)$ for all $x \in X$ and $t \in\left(0, t_{0}\right]$. Fix $x \in X$. Suppose that there is some $c>0$ so that $\frac{1}{t} \mathrm{dvol}_{h(t)}(x) \geq \frac{c}{t_{0}} \mathrm{dvol}_{h\left(t_{0}\right)}$ for all $t \in\left(0, t_{0}\right]$. Then as $s \rightarrow 0$, the rescaled Einstein flows $\mathcal{E}_{s}$, pointed at $x$, approach $\mathcal{K}$ in the pointed weak $W^{2, p}$-topology and the pointed $C^{1, \alpha}$-topology.

We note that even if there is pointwise convergence to a Kasner solution, the Kasner solution can definitely depend on $x$. Besides the obvious example of a Kasner solution 
(Example 4.5), examples of Theorem 1.6 arise from Kantowski-Sachs solutions (Example 4.8), Taub-NUT solutions (Example 4.10), Bianchi VIII NUT solutions (Example 4.11) and, hypothetically, polarized Gowdy spacetimes (Example 4.12).

From [20, Lemma 1], if the Einstein flow has nonpositive spatial scalar curvature then the volume is $O(t)$ as $t \rightarrow 0$. In this case, Theorem 1.6 was proven in 20, Proposition 17]. Also, if the Einstein flow has nonpositive spatial scalar curvature then an integral version of Theorem 1.6 was proven in [20, Proposition 10], without any further curvature assumptions.

Theorems 1.4 and 1.6 roughly describe two extremes of volume shrinkage as $t \rightarrow 0$, namely when the spatial volume density goes like $t^{n}$ or $t$. Some relevant spacetimes for the BKL conjectures are those whose volume densities may be $O(t)$, but are not $O\left(t^{1+\beta}\right)$ for any $\beta>0$. The next theorem roughly says that as one approaches the singularity in such a spacetime, almost all of the time is spent in Kasner-like regions.

Theorem 1.7. Suppose that $\mathcal{E}$ is a type-I CMC Einstein flow with asymptotically nonpositive spatial scalar curvature. Fix $x \in X$. Suppose that for each $\beta>0, \operatorname{dvol}_{h(t)}(x)$ fails to be $O\left(t^{1+\beta}\right)$ as $t \rightarrow 0$. Given $\epsilon>0$, let $S_{\epsilon}$ be the set of $\tau \geq 0$ so that $\mathcal{E}_{t_{0} e^{-\tau}}$, pointed at $x$, is not $\epsilon$-close to $\mathcal{K}$ in the pointed $C^{1, \alpha}$-topology. Given $N \in \mathbb{Z}^{+}$, let $F(N)$ be the number of unit intervals $\{[k, k+1]\}_{k=0}^{N-1}$ that have a nonempty intersection with $S_{\epsilon}$. Then

$$
\liminf _{N \rightarrow \infty} \frac{F(N)}{N}=0
$$

Examples of Theorem 1.7 come from Mixmaster solutions of Bianchi type IX (Example 4.16) and Bianchi type VIII (Example 4.32). One sees in the Mixmaster examples that the particular Kasner-like geometries that are approached can vary wildly as $\tau \rightarrow \infty$.

Regarding the general applicability of Theorems 1.4, 1.6 and 1.7, we do not know an example of a vacuum spacetime, with a crushing singularity, that fails to be type-I with asymptotically nonpositive spatial scalar curvature. However, we have no reason to think that such examples do not exist. One feature of Theorems [1.4, 1.6 and 1.7 is that the volume assumptions are pointwise at $x \in X$. (More geometrically, we are looking at a trajectory that goes backward in time starting from $\left(t_{0}, x\right)$ and is normal to the spatial slices.) This is perhaps consistent with point (1) of the BKL conjectures, at least for Theorems 1.6 and 1.7, where particle horizons can form.

The proofs of Theorems 1.4, 1.6 and 1.7 are based on compactness arguments for Einstein flows, as initiated in [1], and pointwise monotonicity formulas. For Theorem [1.4, the monotonicity formula is from [12]. For Theorems 1.6] and 1.7, the formula is from [20].

The structure of the paper is as follows. In Section 2 we recall background material. In Section 3 we prove Theorems 1.4, 1.6 and 1.7, first under a noncollapsing assumption. In this case, the spaces $\mathcal{M}$ and $\mathcal{K}$ consists of Einstein flows in the usual sense. In Subsection 3.4 we indicate how to remove the noncollapsing assumption, at the price of considering Einstein flows on a more general type of space. Section 4 has examples of spacetimes that satisfy the hypotheses of Theorems 1.4, 1.6 and 1.7, including Mixmaster examples.

I thank the referees for their comments. 


\section{CMC Einstein FlOWS}

In this section we recall some material about CMC Einstein flows, rescalings and convergent subsequences.

\subsection{CMC spacetimes.}

Definition 2.1. Let $I$ be an interval in $\mathbb{R}$. An Einstein flow $\mathcal{E}$ on an $n$-dimensional manifold $X$ is given by a family of nonnegative functions $\{L(t)\}_{t \in I}$ on $X$, a family of Riemannian metrics $\{h(t)\}_{t \in I}$ on $X$, and a family of symmetric covariant 2-tensor fields $\{K(t)\}_{t \in I}$ on $X$, so that if $H=h^{i j} K_{i j}$ and $K^{0}=K-\frac{H}{n} h$ then the constraint equations

$$
R-\left|K^{0}\right|^{2}+\left(1-\frac{1}{n}\right) H^{2}=0
$$

and

$$
\nabla_{i} K_{j}^{i}-\nabla_{j} H=0
$$

are satisfied, along with the evolution equations

$$
\frac{\partial h_{i j}}{\partial t}=-2 L K_{i j}
$$

and

$$
\frac{\partial K_{i j}}{\partial t}=L H K_{i j}-2 L h^{k l} K_{i k} K_{l j}-L_{; i j}+L R_{i j}
$$

For now, we will assume that all of the data is smooth. At the moment, $L$ is unconstrained; it will be determined by the elliptic equation (2.8) below. We will generally want $L(t)$ to be positive.

An Einstein flow gives rise to a Ricci-flat Lorentzian metric

$$
g=-L^{2} d t^{2}+h(t)
$$

on $I \times X$, for which the second fundamental form of the time- $t$ slice is $K(t)$. On the other hand, given a Lorentzian metric $g$ on a manifold with a proper time function $t$, we can write it in the form (2.6) by using curves that meet the level sets orthogonally to give diffeomorphisms between level sets and establish a product structure. Letting $K(t)$ be the second fundamental form of the time- $t$ slice, the metric $g$ is Ricci-flat if and only if $(L, h, K)$ is an Einstein flow.

Definition 2.7. A CMC Einstein flow is an Einstein flow for which $H$ only depends on $t$.

In the definition of a CMC Einstein flow, we do not assume that $X$ is compact. We are interested in the expanding case, so we assume that we have a CMC Einstein flow with $I=\left(0, t_{0}\right]$ (or $\left.I=\left(0, t_{0}\right)\right)$, and that $H$ is monotonically increasing in $t$ and takes all values in $\left(-\infty, H_{0}\right)$ for some $H_{0}<0$. Important examples arise from crushing singularities as $t \rightarrow 0$, in which case $X$ is compact by definition. 
Returning to general expanding CMC Einstein flows, equation (2.5) gives

$$
\begin{aligned}
\frac{\partial H}{\partial t} & =-\triangle_{h} L+L H^{2}+L R \\
& =-\triangle_{h} L+L\left|K^{0}\right|^{2}+\frac{1}{n} L H^{2} .
\end{aligned}
$$

Assuming bounded spatial curvature and bounded $L$ on compact time intervals, the maximum principle gives

$$
L(t) \leq \frac{n}{H^{2}} \frac{\partial H}{\partial t}
$$

There is a pointwise identity

$$
\frac{\partial}{\partial t}\left((-H)^{n} \operatorname{dvol}_{h}\right)=(-H)^{n+1}\left(L-\frac{n}{H^{2}} \frac{\partial H}{\partial t}\right) \operatorname{dvol}_{h} .
$$

From (2.9), it follows that $(-H)^{n} \operatorname{dvol}_{h(t)}$ is pointwise monotonically nonincreasing in $t$.

Also,

$$
\frac{\partial}{\partial t}\left((-H) \mathrm{dvol}_{h}\right)=H^{2}\left(L-\frac{1}{H^{2}} \frac{\partial H}{\partial t}\right) \mathrm{dvol}_{h} .
$$

2.2. Rescaling limits. Let $\mathcal{E}$ be an Einstein flow. Let $g$ be the corresponding Lorentzian metric. For $s>0$, the Lorentzian metric $s^{-2} g$ is isometric to

$$
g_{s}=-L^{2}(s u) d u^{2}+s^{-2} h(s u) .
$$

Hence we put

$$
\begin{array}{lc}
L_{s}(u)=L(s u), & h_{s}(u)=s^{-2} h(s u), \quad K_{s, i j}(u)=s^{-1} K_{i j}(s u), \\
H_{s}(u)=s H(s u), & K_{s, i j}^{0}(u)=s^{-1} K_{i j}^{0}(s u), \quad\left|K^{0}\right|_{s}^{2}(u)=s^{2} K_{i j}(s u), \\
R_{s, i j}(u)=R_{i j}(s u), & R_{s}(u)=s^{2} R(s u) .
\end{array}
$$

The variable $u$ will refer to the time parameter of a rescaled Einstein flow, or a limit of such. We write the rescaled Einstein flow as $\mathcal{E}_{s}$.

Put $e_{0}=T=\frac{1}{L} \frac{\partial}{\partial t}$, a unit timelike vector that is normal to the level sets of $t$. Let $\left\{e_{i}\right\}_{i=1}^{n}$ be an orthonormal basis for $e_{0}^{\perp}$. Put

$$
|\mathrm{Rm}|_{T}=\sqrt{\sum_{\alpha, \beta, \gamma, \delta=0}^{n} R_{\alpha \beta \gamma \delta}^{2}} .
$$

Hereafter we assume that our CMC Einstein flows have complete spatial slices, and that $|\mathrm{Rm}|_{T}$ is bounded on compact time intervals.

Let $\mathcal{E}^{\infty}=\left(L^{\infty}, h^{\infty}, K^{\infty}\right)$ be a CMC Einstein flow on a pointed $n$-manifold $\left(X^{\infty}, x^{\infty}\right)$, defined on a time interval $I^{\infty}$. Take $p \in[1, \infty)$ and $\alpha \in(0,1)$.

Definition 2.15. The Einstein flow $\mathcal{E}^{\infty}$ is $W^{2, p}$-regular if $X^{\infty}$ is a $W^{3, p}$-manifold, $L^{\infty}$ and $h^{\infty}$ are locally $W^{2, p}$-regular in space and time, and $K^{\infty}$ is locally $W^{1, p}$-regular in space and time. 
Note that the equations of Definition 2.1 make sense in this generality.

Let $\mathcal{E}^{(k)}=\left\{h^{(k)}, K^{(k)}, L^{(k)}\right\}_{k=1}^{\infty}$ be smooth CMC Einstein flows on pointed $n$-manifolds $\left\{\left(X^{(k)}, x^{(k)}\right)\right\}_{k=1}^{\infty}$, defined on time intervals $I^{(k)}$.

Definition 2.16. We say that $\lim _{k \rightarrow \infty} \mathcal{E}^{(k)}=\mathcal{E}^{\infty}$ in the pointed weak $W^{2, p}$-topology if

- Any compact interval $S \subset I^{\infty}$ is contained in $I^{(k)}$ for large $k$, and

- For any compact interval $S \subset I^{\infty}$ and any compact $n$-dimensional manifold-withboundary $W^{\infty} \subset X^{\infty}$ containing $x^{\infty}$, for large $k$ there are pointed time-independent $W^{3, p}$-regular diffeomorphisms $\phi_{S, W^{\infty}, k}: W^{\infty} \rightarrow W^{(k)}$ (with $\left.W^{(k)} \subset X^{(k)}\right)$ so that

$-\lim _{k \rightarrow \infty}\left(\text { Id. } \times \phi_{S, W^{\infty}, k}\right)^{*} L^{(k)}=L^{\infty}$ weakly in $W^{2, p}$ on $S \times W^{\infty}$,

- $\lim _{k \rightarrow \infty}\left(\text { Id. } \times \phi_{S, W^{\infty}, k}\right)^{*} h^{(k)}=h^{\infty}$ weakly in $W^{2, p}$ on $S \times W^{\infty}$ and

$-\lim _{k \rightarrow \infty}\left(\text { Id. } \times \phi_{S, W^{\infty}, k}\right)^{*} K^{(k)}=K^{\infty}$ weakly in $W^{1, p}$ on $S \times W^{\infty}$.

We define pointed (norm) $C^{1, \alpha}$-convergence similarly.

Definition 2.17. Let $\mathcal{S}$ be a collection of pointed CMC Einstein flows defined on a time interval $I^{\infty}$. We say that a sequence $\left\{\mathcal{E}^{(k)}\right\}_{k=1}^{\infty}$ of pointed $C M C$ Einstein flows approaches $\mathcal{S}$ as $k \rightarrow \infty$, in the pointed weak $W^{2, p}$-topology, if for any subsequence of $\left\{\mathcal{E}^{(k)}\right\}_{k=1}^{\infty}$, there is a further subsequence that converges to an element of $\mathcal{S}$ in the pointed weak $W^{2, p}$-topology.

Definition 2.18. Let $\mathcal{S}$ be a collection of pointed $C M C$ Einstein flows defined on a time interval $I^{\infty}$. We say that a 1-parameter family $\left\{\mathcal{E}^{(s)}\right\}_{s \in\left(0, s_{0}\right]}$ of pointed $C M C$ Einstein flows approaches $\mathcal{S}$ as $s \rightarrow 0$, in the pointed weak $W^{2, p}$-topology, if for any sequence $\left\{s_{k}\right\}_{k=1}^{\infty}$ in $\left(0, s_{0}\right]$ with $\lim _{k \rightarrow \infty} s_{k}=0$, there is a subsequence of the Einstein flows $\left\{\mathcal{E}^{\left(s_{k}\right)}\right\}_{k=1}^{\infty}$ that converges to an element of $\mathcal{S}$ in the pointed weak $W^{2, p}$-topology.

We define "approaches $\mathcal{S}$ " in the pointed (norm) $C^{1, \alpha}$-topology similarly. The motivation for these definitions comes from how one can define convergence to a compact subset of a metric space, just using the notion of sequential convergence. In our applications, the relevant set $\mathcal{S}$ of Einstein flows can be taken to be sequentially compact.

Definition 2.19. We say that a pointed $C M C$ Einstein flow $\mathcal{E}^{1}$ is $\epsilon$-close to a pointed $C M C$ Einstein flow $\mathcal{E}^{2}$ in the pointed $C^{1, \alpha}$-topology if they are both defined on the time interval $\left(\epsilon, \epsilon^{-1}\right)$ and, up to applying time-independent pointed diffeomorphisms, the metrics are $\epsilon$-close in the $C^{1, \alpha}$-norm on $\left(\epsilon, \epsilon^{-1}\right) \times B_{h_{2}(1)}\left(x^{(2)}, \epsilon^{-1}\right)$.

We don't make a similar definition of closeness for the pointed weak $W^{2, p}$-topology because the weak topology is not metrizable.

We now take $t=-\frac{n}{H}$, with $t$ ranging in an interval $\left(0, t_{0}\right]$.

Definition 2.20. A type-I Einstein flow is a CMC Einstein flow for which there is some $C<\infty$ so that $|\mathrm{Rm}|_{T} \leq C t^{-2}$ for all $t \in\left(0, t_{0}\right]$.

Let $B_{h(t)}(x, t)$ denote the time- $t$ metric ball of radius $t$ around $x$.

Definition 2.21. If $\mathcal{E}$ is a $C M C$ Einstein flow and $x \in X$ then the flow is noncollapsing at $x$ as $t \rightarrow 0$ if $\operatorname{vol}\left(B_{h(t)}(x, t)\right) \geq v_{0} t^{n}$ for all $t$, for some $v_{0}>0$. 
We do not know examples of crushing singularities for which the Einstein flow fails to be everywhere noncollapsing.

Proposition 2.22. [20, Proposition 11] Let $\mathcal{E}$ be a type-I Einstein flow on an $n$-dimensional manifold $X$. Suppose that it is defined on a time-interval $\left(0, t_{0}\right]$ and has complete time slices. Suppose that it is noncollapsing at $x \in X$ as $t \rightarrow 0$. Given a sequence $s_{i} \rightarrow 0$, after passing to a subsequence, there is a limit $\lim _{i \rightarrow \infty} \mathcal{E}_{s_{i}}=\mathcal{E}^{\infty}$ in the pointed weak $W^{2, p_{-}}$ topology and the pointed $C^{1, \alpha}$-topology. The limit Einstein flow $\mathcal{E}^{\infty}$ is defined on the time interval $(0, \infty)$. Its time slices $\left\{\left(X^{\infty}, h^{\infty}(u)\right)\right\}_{u>0}$ are complete. Its lapse function $L^{\infty}$ is uniformly bounded below by a positive constant.

\section{Asymptotic Geometry}

In this section we prove Theorems [1.4, 1.6 and 1.7. We initially prove them in the noncollapsing case. In Subsection 3.4 we indicate how to remove this assumption.

3.1. Milne asymptotics. Let $\mathcal{M}$ be the collection of pointed Einstein flows that describe Lorentzian cones over pointed Riemannian Einstein $n$-manifolds with Einstein constant $-(n-1)$. We take the basepoint for such a flow to be at time one. The proof of the next proposition is similar to that of [19, Proposition 3.5].

Proposition 3.1. Suppose that $\mathcal{E}$ is a type-I CMC Einstein flow. Fix $x \in X$. Suppose that $\mathcal{E}$ is noncollapsing at $x$ and $\operatorname{dvol}_{h(t)}(x)$ is $O\left(t^{n}\right)$ as $t \rightarrow 0$. Then as $s \rightarrow 0$, the rescaled Einstein flows $\mathcal{E}_{s}$, pointed at $x$, approach $\mathcal{M}$ in the pointed weak $W^{2, p}$-topology and the pointed $C^{1, \alpha}$-topology.

Proof. Suppose that the claim is not true. Let $\left\{s_{i}\right\}_{i=1}^{\infty}$ be a sequence with $\lim _{i \rightarrow \infty} s_{i}=0$ such that no subsequence of $\left\{\mathcal{E}_{s_{i}}\right\}_{i=1}^{\infty}$ converges to an element of $\mathcal{M}$ in the given topologies. From (2.9), we have $L \leq 1$. From (2.10), $t^{-n} \mathrm{dvol}_{h(t)}(x)$ is monotonically nonincreasing in $t$, and

$$
\log \frac{t^{-n} \operatorname{dvol}_{h(t)}(x)}{t_{0}^{-n} \operatorname{dvol}_{h\left(t_{0}\right)}(x)}=n \int_{t}^{t_{0}}(1-L(v, x)) \frac{d v}{v} .
$$

By assumption, $t^{-n} \operatorname{dvol}_{h(t)}(x)$ is uniformly bounded above, so

$$
\int_{0}^{t_{0}}(1-L(v, x)) \frac{d v}{v}<\infty .
$$

From Proposition 2.22, after passing to a subsequence, we can assume that $\lim _{i \rightarrow \infty} \mathcal{E}_{s_{i}}=$ $\mathcal{E}^{\infty}$ for a pointed CMC Einstein flow $\mathcal{E}^{\infty}$. As the inequality $L \leq 1$ passes to the limit, we know that $L^{\infty} \leq 1$. We claim that $L^{\infty}\left(u, x^{\infty}\right)=1$ for all $u \in(0, \infty)$. If not then $L^{\infty}\left(\widehat{u}, x^{\infty}\right) \leq 1-\delta$ for some $\delta>0$ and $\widehat{u} \in(0, \infty)$. By continuity, there is some $\mu>0$ so that $L^{\infty}\left(\widehat{u} e^{\bar{\sigma}}, x^{\infty}\right) \leq 1-\frac{\delta}{2}$ for all $\sigma \in[-\mu, \mu]$. Then for sufficiently large $i$, we know that $L\left(s_{i} \widehat{u} e^{\sigma}, x\right) \leq 1-\frac{\delta}{4}$ for all $\sigma \in[-\mu, \mu]$. After passing to a subsequence, we can assume that the intervals $\left\{\left[s_{i} \widehat{u} e^{-\mu}, s_{i} \widehat{u} e^{\mu}\right]\right\}_{i=1}^{\infty}$ are disjoint. We obtain a contradiction to (3.3).

Thus $L^{\infty}\left(u, x^{\infty}\right)=1$ for all $u \in(0, \infty)$. Equation (2.8) (with $t$ replaced by $u$ ), along with elliptic regularity, the fact that $h^{\infty}$ is locally $C^{1, \alpha}$-regular and the fact that $\left(K^{\infty}\right)^{0}$ is locally 
$C^{\alpha}$-regular, implies that $L^{\infty}(u, \cdot)$ is locally $C^{2, \alpha}$-regular. We can apply the strong maximum principle to (2.8) on $X^{\infty}$ to obtain that $L^{\infty}=1$ and $\left(K^{\infty}\right)^{0}=0$. As $K^{\infty}(u)=-\frac{1}{u} h^{\infty}(u)$, it follows from (2.4) that $h^{\infty}(c u)=c^{2} h^{\infty}(u)$. Then (2.5) implies that $h^{\infty}(1)$ is an Einstein manifold with Einstein constant $-(n-1)$. Hence there is a subsequence of $\left\{\mathcal{E}_{s_{i}}\right\}_{i=1}^{\infty}$ that converges to an element of $\mathcal{M}$, which is a contradiction.

\subsection{Kasner-like asymptotics.}

Lemma 3.4. Given a function $\widehat{R}$ of $t$, if $R(t, x) \leq \widehat{R}(t)$ for all $x \in X$ then $L(t, x) \geq$ $\frac{n}{n^{2}+t^{2} \widehat{R}(t)}$ for all $x \in X$, as long as the denominator is positive.

Proof. This follows from applying the weak maximum principle to (2.8).

Let $\mathcal{K}$ be the collection of pointed expanding CMC Einstein flows with $R=0, L=\frac{1}{n}$ and $|K|^{2}=H^{2}$, defined for $t \in(0, \infty)$. We take the basepoint for such a flow to be at time one.

Proposition 3.5. Let $\mathcal{E}$ be a type-I CMC Einstein flow with asymptotically nonpositive spatial scalar curvature. Suppose in addition that there is a nonnegative function $\widehat{R}$ : $\left(0, t_{0}\right] \rightarrow \mathbb{R}$ with $\int_{0}^{t_{0}} t^{2} \widehat{R}(t) \frac{d t}{t}<\infty$ so that $R(x, t) \leq \widehat{R}(t)$ for all $x \in X$ and $t \in\left(0, t_{0}\right]$. Fix $x \in X$. Suppose that $\mathcal{E}$ is noncollapsing at $x$ and there is some $c>0$ so that $\frac{1}{t} \operatorname{dvol}_{h(t)}(x) \geq$ $\frac{c}{t_{0}} \operatorname{dvol}_{h\left(t_{0}\right)}$ for all $t \in\left(0, t_{0}\right]$. Then as $s \rightarrow 0$, the rescaled Einstein flows $\mathcal{E}_{s}$, pointed at $x$, approach $\mathcal{K}$ in the pointed weak $W^{2, p}$-topology and the pointed $C^{1, \alpha}$-topology.

Proof. Suppose that the claim fails. Then there is a sequence $s_{i} \rightarrow 0$ with the property that no subsequence of $\mathcal{E}_{s_{i}}$ converges to an element of $\mathcal{K}$ in the given topologies.

After passing to a subsequence, we can assume that $\lim _{i \rightarrow \infty} \mathcal{E}_{s_{i}}=\mathcal{E}^{\infty}$ for a CMC Einstein flow $\mathcal{E}^{\infty}$.

Lemma 3.6. $L^{\infty}\left(u, x^{\infty}\right) \leq \frac{1}{n}$ for all $u \in(0, \infty)$.

Proof. Suppose that $L^{\infty}\left(u, x^{\infty}\right) \geq \frac{1}{n}+\delta$ for some $u \in(0, \infty)$ and some $\delta>0$. By continuity, there is some $\mu$ so that $L^{\infty}\left(u e^{\sigma}, x^{\infty}\right) \geq \frac{1}{n}+\frac{1}{2} \delta$ for all $\sigma \in[-\mu, \mu]$. Then for large $i$, we know that $L\left(s_{i} u e^{\sigma}, x\right) \geq \frac{1}{n}+\frac{1}{4} \delta$ for all $\sigma \in[-\mu, \mu]$. After passing to a subsequence, we can assume that the intervals $\left\{\left[s_{i} u e^{-\mu}, s_{i} u e^{\mu}\right]\right\}_{i=1}^{\infty}$ are disjoint. From Lemma $\underline{3.4}$,

$$
L(v, x)-\frac{1}{n} \geq \frac{n}{n^{2}+v^{2} \widehat{R}(v)}-\frac{1}{n}=-\frac{v^{2} \widehat{R}(v)}{n\left(n^{2}+v^{2} \widehat{R}(v)\right)} \geq-\frac{v^{2} \widehat{R}(v)}{n^{3}} .
$$

Hence the negative part of $L(v, x)-\frac{1}{n}$ is integrable with respect to $\frac{d v}{v}$. It follows that

$$
\int_{0}^{t_{0}}\left(L(v, x)-\frac{1}{n}\right) \frac{d v}{v}=\infty .
$$

However, from (2.11), we have

$$
\log \frac{\frac{1}{t_{0}} \operatorname{dvol}_{h\left(t_{0}\right)}(x)}{\frac{1}{t} \operatorname{dvol}_{h(t)}(x)}=n \int_{t}^{t_{0}}\left(L(v, x)-\frac{1}{n}\right) \frac{d v}{v} .
$$


By our assumptions, the left-hand side of (3.9) is bounded as $t \rightarrow 0$. This is a contradiction, so $L^{\infty}\left(u, x^{\infty}\right) \leq \frac{1}{n}$ for all $u \in(0, \infty)$.

Lemma 3.10. $R^{\infty} \leq 0$.

Proof. Note that $R^{\infty} \in L_{l o c}^{p}\left((0, \infty) \times X^{\infty}\right)$. Let $f$ be a compactly supported nonnegative continuous function on $(0, \infty) \times X^{\infty}$. Choose a compact interval $S \subset(0, \infty)$ and a compact $n$-dimensional manifold-with-boundary $W^{\infty} \subset X^{\infty}$ so that the support of $f$ is contained in $S \times W^{\infty}$. With reference to Definition 2.16, the weak $L^{p}$-convergence of scalar curvature gives

$$
\int_{S} \int_{X^{\infty}} f R^{\infty} \operatorname{dvol}_{h^{\infty}(u)} u^{2} d u=\lim _{i \rightarrow \infty} \int_{S} \int_{X^{\infty}} f\left(\mathrm{Id} . \times \phi_{S, W^{\infty}, i}\right)^{*} R_{s_{i}} \operatorname{dvol}_{h^{\infty}(u)} u^{2} d u .
$$

Since $\mathcal{E}$ has asymptotically nonpositive spatial scalar curvature, we can assume that $\lim _{t \rightarrow 0} t^{2} \widehat{R}(t)=0$. Now

$$
\begin{aligned}
& \int_{S} \int_{X^{\infty}} f\left(\operatorname{Id} . \times \phi_{S, W^{\infty}, i}\right)^{*} R_{s_{i}} \operatorname{dvol}_{h^{\infty}(u)} u^{2} d u \leq \\
& \left(\int_{S} \int_{X^{\infty}} f \operatorname{dvol}_{h^{\infty}(u)} d u\right) \max _{u \in S} s_{i}^{2} u^{2} \widehat{R}\left(s_{i} u\right) .
\end{aligned}
$$

Hence

$$
\int_{S} \int_{X^{\infty}} f R^{\infty} \operatorname{dvol}_{h^{\infty}(u)} u^{2} d u \leq 0
$$

for all such $f$, which proves the lemma.

Lemma 3.14. $\mathcal{E}^{\infty} \in \mathcal{K}$.

Proof. From Lemma 3.4 and the asymptotic nonpositive spatial scalar curvature, we know that $L^{\infty}\left(u, x^{\infty}\right) \geq \frac{1}{n}$ for all $u \in(0, \infty)$. Then Lemma 3.6 implies that $L^{\infty}\left(u, x^{\infty}\right)=\frac{1}{n}$ for all $u \in(0, \infty)$.. Fix $u$. By the same argument as in the proof of Proposition 3.1, we know that $L^{\infty}(u, \cdot)$ is locally $C^{2, \alpha}$-regular. Then we can apply the strong maximum principle to (2.8) to conclude that $L^{\infty}=\frac{1}{n}$. From (2.8) again, we obtain $R^{\infty}=0$. (Note that from the constraint equation (2.2), $R^{\infty}$ is locally Hölder-continuous.) Then (2.2) gives $\left|K^{\infty}\right|^{2}=H^{2}$. Thus $\mathcal{E}^{\infty} \in \mathcal{K}$.

We have found a subsequence of $\left\{\mathcal{E}_{s_{i}}\right\}_{i=1}^{\infty}$ that converges to an element of $\mathcal{K}$, which is a contradiction. This proves the proposition.

\subsection{Kasner-like time intervals.}

Proposition 3.15. Suppose that $\mathcal{E}$ is a type-I CMC Einstein flow with asymptotically nonpositive spatial scalar curvature. Fix $x \in X$. Suppose that $\mathcal{E}$ is noncollapsing at $x$ and for each $\beta>0, \operatorname{dvol}_{h(t)}(x)$ fails to be $O\left(t^{1+\beta}\right)$ as $t \rightarrow 0$. Given $\epsilon>0$, let $S_{\epsilon}$ be the set of $\tau \geq 0$ so that $\mathcal{E}_{t_{0} e^{-\tau}}$, pointed at $x$, is not $\epsilon$-close to $\mathcal{K}$ in the pointed $C^{1, \alpha}$-topology. Given 
$N \in \mathbb{Z}^{+}$, let $F(N)$ be the number of unit intervals $\{[k, k+1]\}_{k=0}^{N-1}$ that have a nonempty intersection with $S_{\epsilon}$. Then

$$
\liminf _{N \rightarrow \infty} \frac{F(N)}{N}=0
$$

Proof. We begin with a couple of lemmas.

Lemma 3.17. Given $\epsilon>0$, there is a $\delta>0$ so that if $\tau \geq 2 \delta^{-1}$, and $L\left(t_{0} e^{-\tau^{\prime}}, x\right) \leq \frac{1}{n}+\delta$ for $\tau^{\prime} \in\left[\tau-\delta^{-1}, \tau+\delta^{-1}\right]$, then $\mathcal{E}_{t_{0} e^{-\tau}}$ is $\epsilon$-close to $\mathcal{K}$ in the pointed $C^{1, \alpha}$-topology.

Proof. If the lemma is not true then there is a sequence $\delta_{i} \rightarrow 0$ and for each $i$, some $\tau_{i} \geq 2 \delta_{i}^{-1}$ so that $L\left(t_{0} e^{-\tau^{\prime}}, x\right) \leq \frac{1}{n}+\delta_{i}$ for $\tau^{\prime} \in\left[\tau_{i}-\delta_{i}^{-1}, \tau_{i}+\delta_{i}^{-1}\right]$, but $\mathcal{E}_{t_{0} e^{-\tau_{i}}}$ is not $\epsilon$-close to $\mathcal{K}$ in the pointed $C^{1, \alpha}$-topology. After passing to a subsequence, we can assume that $\lim _{i \rightarrow \infty} \mathcal{E}_{t_{0} e^{-\tau_{i}}}=\mathcal{E}^{\infty}$ in the pointed $C^{1, \alpha}$-topology, for some CMC Einstein flow $\mathcal{E}^{\infty}$ defined for time parameter $u \in(0, \infty)$. By construction, $L^{\infty}\left(u, x^{\infty}\right) \leq \frac{1}{n}$ for all $u \in(0, \infty)$. As $\mathcal{E}$ has asymptotically nonpositive spatial scalar curvature, the proof of Lemma 3.10 shows that $R^{\infty} \leq 0$. The proof of Lemma 3.14 applies again, so $\mathcal{E}^{\infty} \in \mathcal{K}$. Hence $\mathcal{E}_{t_{0} e^{-\tau_{i}}}$ is $\epsilon$-close to $\mathcal{K}$ for large $i$, which is a contradiction.

Lemma 3.18. Given $\delta>0$, there are $t^{\prime}, \mu>0$ with the following property. Suppose that $t \leq t^{\prime}$ and $L(t, x)>\frac{1}{n}+\delta$. Then $L\left(t e^{\sigma}, x\right)>\frac{1}{n}+\frac{1}{2} \delta$ for $\sigma \in[-\mu, \mu]$.

Proof. If the claim is not true then taking $\mu_{i}=\frac{1}{i}$, there is a sequence $\left\{t_{i, j}\right\}_{j=1}^{\infty}$ with $\lim _{j \rightarrow \infty} t_{i, j}=0$ so that $L\left(t_{i, j}, x\right)>\frac{1}{n}+\delta$, but $L\left(t_{i, j} e^{\sigma}, x\right) \leq \frac{1}{n}+\frac{1}{2} \delta$ for some $\sigma \in\left[-\mu_{i}, \mu_{i}\right]$. Passing to a diagonal subsequence, there are sequences $\mu_{i} \rightarrow 0$ and $t_{i} \rightarrow 0$ so that $L\left(t_{i}, x\right)>$ $\frac{1}{n}+\delta$ but $L\left(t_{i} e^{\sigma}, x\right) \leq \frac{1}{n}+\frac{1}{2} \delta$ for some $\sigma \in\left[-\mu_{i}, \mu_{i}\right]$. Passing to a subsequence, we can assume that $\lim _{i \rightarrow \infty} \mathcal{E}_{t_{i}}=\mathcal{E}^{\infty}$ for some CMC Einstein flow $\mathcal{E}^{\infty}$. Then $L^{\infty}\left(1, x^{\infty}\right) \geq \frac{1}{n}+\delta$. By continuity, there is some $\mu^{\prime}>0$ so that $L^{\infty}\left(e^{\sigma}, x^{\infty}\right) \geq \frac{1}{n}+\frac{8}{9} \delta$ for all $\sigma \in\left[-\mu^{\prime}, \mu^{\prime}\right]$. Then for large $i$, we know that $L\left(t_{i} e^{\sigma}, x\right) \geq \frac{1}{n}+\frac{3}{4} \delta$ for all $\sigma \in\left[-\mu^{\prime}, \mu^{\prime}\right]$. This is a contradiction.

To prove the proposition, suppose that it is not true. Then there is some $c>0$ so that $F(N) \geq c N$ for large $N$.

Let $\delta$ be the parameter from Lemma 3.17. For $N \in \mathbb{Z}^{+}$, let $G(N)$ be the number of unit intervals $\{[k, k+1]\}_{k=0}^{N-1}$ that contain a number $\sigma$ for which $L\left(t_{0} e^{-\sigma}, x\right)>\frac{1}{n}+\delta$. Lemma 3.17 implies that there is some $c^{\prime}>0$ so that $G(N) \geq c^{\prime} N$ for large $N$. Lemmas 3.4 and 3.18 now imply that there is some $c^{\prime \prime}>0$ so that

$$
\int_{t}^{t_{0}}\left(L(v, x)-\frac{1}{n}\right) \frac{d v}{v} \geq c^{\prime \prime} \log \frac{t_{0}}{t}
$$

for small $t$. Equation (3.9) then gives

$$
\operatorname{dvol}_{t}(x) \leq\left(\frac{t}{t_{0}}\right)^{1+n c^{\prime \prime}} \operatorname{dvol}_{t_{0}}(x)
$$

for small $t$, which contradicts the assumptions of the proposition. This proves the proposition. 
3.4. Collapsing case. We indicate how to remove the noncollapsing assumption in Propositions 3.1, 3.5 and 3.15. Without this assumption, we can again take pointed limits of Einstein flows but the limit flow will generally be on an étale groupoid instead of a manifold. For background information on étale groupoids and Einstein flows on étale groupoids, we refer to [18, Section 5] and [19, Section 3.1]. We can define $\epsilon$-closeness of Einstein flows on étale groupoids in the $C^{1, \alpha}$-topology analogously to Definition 2.19, using the setup of [18, Definition 5.8]. The strong maximum principle applies on the unit space of the groupoid directly.

We define $\mathcal{M}$ and $\mathcal{K}$ as in Subsections 3.1 and 3.2, except with the Einstein flows being on étale groupoids, Then the proofs of Propositions 3.1, 3.5 and 3.15 go through without significant change.

\section{EXAMPLES}

Example 4.1. Consider a Lorentzian cone over a Riemannian Einstein $n$-manifold $\left(X, h_{\text {Ein }}\right)$ with Einstein constant $-(n-1)$. The metric is

$$
g=-d t^{2}+t^{2} h_{\text {Ein }}
$$

The corresponding Einstein flow $\mathcal{E}$ is a type-I CMC Einstein flow. It is noncollapsing at each $x \in X$ as $t \rightarrow 0$. The volume density $\operatorname{dvol}_{h(t)}(x)$ is proportionate to $t^{n}$. The pointed rescaling limit $\lim _{s \rightarrow 0} \mathcal{E}_{s}$ equals $\mathcal{E}$. This gives an example of Proposition 3.1 .

Example 4.3. Consider the product of the previous example, in dimension $n-n^{\prime}$, with a flat torus $\left(T^{n^{\prime}}, h_{\text {flat }}\right)$. The metric is

$$
g=-d t^{2}+t^{2} h_{\text {Ein }}+h_{\text {flat }} .
$$

The corresponding Einstein flow $\mathcal{E}$ is a type-I CMC Einstein flow and has nonpositive spatial scalar curvature. It is noncollapsing at each $x \in X$ as $t \rightarrow 0$. The volume density $\operatorname{dvol}_{h(t)}(x)$ is proportionate to $t^{n-n^{\prime}}$.

The pointed rescaling limit $\lim _{s \rightarrow 0} \mathcal{E}_{s}$ is the product of the Einstein flow of the previous example with flat $\mathbb{R}^{n^{\prime}}$. If $n-n^{\prime}=1$ then we get an example of Proposition 3.5.

Example 4.5. Consider a Kasner solution on a flat $n$-manifold. After possibly passing to a cover of $X$, the metric is

$$
g=-\frac{1}{n^{2}} d t^{2}+(d \vec{x})^{T} t^{2 M} d \vec{x}
$$

Here $M$ is a symmetric $(n \times n)$-matrix with $\operatorname{Tr}(M)=\operatorname{Tr}\left(M^{2}\right)=1$. We have written the metric so that $t=-\frac{n}{H}$. Then

$$
L=\frac{1}{n}, \quad R=0, \quad|K|^{2}=H^{2}=\frac{n^{2}}{t^{2}} .
$$

The corresponding Einstein flow $\mathcal{E}$ is a type-I CMC Einstein flow and has vanishing spatial scalar curvature. It is noncollapsing at each $x \in X$ as $t \rightarrow 0$. The volume density $\operatorname{dvol}_{h(t)}(x)$ is proportionate to $t$. 
The pointed rescaling limit $\lim _{s \rightarrow 0} \mathcal{E}_{s}$ is a Kasner flow, with the same matrix $M$. It lives on $\mathbb{R}^{n}$ provided that $M$ does not have 1 as an eigenvalue. This gives an example of Proposition 3.5.

Example 4.8. Consider a Kantowski-Sachs solution with $X$ diffeomorphic to $S^{2} \times S^{1}$. The metric is a $\mathbb{Z}$-quotient of the interior of the event horizon in a Schwarzschild solution, after switching the usual $t$ and $r$ variables:

$$
g=-\frac{1}{\frac{2 m}{t}-1} d t^{2}+\left(\frac{2 m}{t}-1\right) d r^{2}+t^{2} g_{S^{2}} .
$$

Here $t \in(0,2 m)$ and the $\mathbb{Z}$-quotienting is in the $r$-variable. The corresponding Einstein flow $\mathcal{E}$ is a type-I CMC Einstein flow, although the parameter $t$ in (4.9) is not the Hubble time $t_{H}$. The relation is that for small time, $t \sim t_{H}^{\frac{2}{3}}$. The spatial slices have scalar curvature $R(t)=\frac{2}{t^{2}}$, which goes like $t_{H}^{-\frac{4}{3}}$. It follows that in terms of the Hubble time, $\int_{0}^{t_{0}} v^{2} R(v) \frac{d v}{v}<\infty$. The Einstein flow is noncollapsing at each $x \in X$ as $t_{H} \rightarrow 0$. The volume density $\operatorname{dvol}_{h\left(t_{H}\right)}(x)$ goes like $t_{H}$. This gives an example of Proposition 3.5. The pointed rescaling limit $\lim _{s \rightarrow 0} \mathcal{E}_{s}$ is the Kasner flow on $\mathbb{R}^{3}$ with a diagonal matrix $M$ whose diagonal entries are $\left\{\frac{2}{3}, \frac{2}{3},-\frac{1}{3}\right\}$.

Example 4.10. Consider an Einstein flow on $S^{3} \cong \mathrm{SU}(2)$ that is left $\mathrm{SU}(2)$-invariant and right U(1)-invariant. This is the Taub part of the Taub-NUT solution [11, Section 9.2.7]. The corresponding Einstein flow is a type-I CMC Einstein flow. The spatial slices have positive scalar curvature but one can check that $\int_{0}^{t_{0}} v^{2} R(v) \frac{d v}{v}<\infty$. The Einstein flow is noncollapsing at each $x \in X$ as $t \rightarrow 0$. The volume density $\operatorname{dvol}_{h\left(t_{H}\right)}(x)$ goes like $t$. This gives an example of Proposition 3.5. Geometrically, before rescaling, the GromovHausdorff limit as $t \rightarrow 0$ is a 2 -sphere and the circle fibers have length that goes like $t$. The pointed rescaling limit $\lim _{s \rightarrow 0} \mathcal{E}_{s}$ is a Kasner flow on $S^{1} \times \mathbb{R}^{2}$ with a diagonal matrix $M$ whose diagonal entries are $\{1,0,0\}$.

Example 4.11. Let $\mathcal{E}$ be a Bianchi-VIII NUT solution on a circle bundle over a higher genus surface [11, Section 9.2.6]. The corresponding Einstein flow is a type-I CMC Einstein flow. The spatial slices have negative scalar curvature. The Einstein flow is noncollapsing at each $x \in X$ as $t \rightarrow 0$. The volume density $\operatorname{dvol}_{h\left(t_{H}\right)}(x)$ goes like $t$. This gives an example of Proposition 3.5. Geometrically, before rescaling, the Gromov-Hausdorff limit as $t \rightarrow 0$ is the surface and the circle fibers have length that goes like $t$. The pointed rescaling limit $\lim _{s \rightarrow 0} \mathcal{E}_{s}$ is a Kasner flow on $S^{1} \times \mathbb{R}^{2}$ with a diagonal matrix $M$ whose diagonal entries are $\{1,0,0\}$.

Example 4.12. Consider a four dimensional polarized Gowdy spacetime with spatial slices diffeomorphic to $T^{3}$ [16]. The metric can be written

$$
g=e^{2 a}\left(-d t^{2}+d \theta^{2}\right)+t\left(e^{W} d x^{2}+e^{-W} d y^{2}\right) .
$$


Here $a$ and $W$ are functions of $t$ and $\theta$. Define $\tau$ by $t=e^{-\tau}$, so one approaches the singularity as $\tau \rightarrow \infty$. Asymptotics as $\tau \rightarrow \infty$ were derived in [16]. In particular,

$$
\begin{aligned}
a(\tau, \theta) & \sim \frac{1-\pi^{2}(\theta)}{4}\left(\tau-\tau_{0}\right)+\alpha(\theta)+\ldots, \\
W(\tau, \theta) & \sim \pi(\theta)\left(\tau-\tau_{0}\right)+\omega(\theta)+\ldots
\end{aligned}
$$

for appropriate functions $\alpha, \pi, \omega$ of $\theta$.

As the singularity is a crushing singularity, there is a CMC foliation, but the metric (4.13) is not in CMC form. Because of this, one cannot read off whether the hypotheses of Proposition 3.5 are fulfilled. Nevertheless, one can say the following. First, from the barrier argument for CMC hypersurfaces [13], one can show that the Hubble time $t_{H}$ goes like $e^{-\frac{\pi^{2}(\theta)+3}{4} \tau}$. Then from [16, Theorem IV.1], the Kretschmann scalar satisfies

$$
\left|R^{\alpha \beta \gamma \delta} R_{\alpha \beta \gamma \delta}\right| \leq \text { const. } t_{H}^{-4},
$$

which is consistent with type-I asymptotics. Next, we can estimate the spacetime volume $V\left(t_{H}\right)$ between the singularity and the CMC slice with $H=-\frac{3}{t_{H}}$. One finds that $V\left(t_{H}\right)$ goes like $t_{H}^{2}$, which is consistent with $\operatorname{dvol}_{h\left(t_{H}\right)}$ going like $t_{H}$.

Fixing $\theta, x$ and $y$, the geometry as $\tau \rightarrow \infty$ approaches a Kasner geometry on $\mathbb{R}^{3}$ with a diagonal matrix $M$ whose diagonal entries are $\left\{\frac{\pi^{2}(\theta)-1}{\pi^{2}(\theta)+3}, \frac{2-2 \pi(\theta)}{\pi^{2}(\theta)+3}, \frac{2+2 \pi(\theta)}{\pi^{2}(\theta)+3}\right\}$.

Example 4.16. Consider an Einstein flow of Bianchi type IX [11, Section 6.4]. It is a homogeneous CMC Einstein flow on $S^{3}$ with a crushing singularity. The Einstein flow is left $\mathrm{SU}(2)$-invariant. The case when it is right $\mathrm{U}(1)$-invariant was already considered in Example 4.10, so we assume that the flow is not right U(1)-invariant, i.e. it is of Mixmaster type.

Proposition 4.17. A Bianchi IX Mixmaster flow is type-I and has asymptotically nonpositive spatial scalar curvature.

Proof. To describe the ODE of the Bianchi IX flow, we use the normalizations of [24]; see [24, Appendix]. The metric can be written as

$$
g=-d t^{2}+h(t)
$$

(The $t$ here is not the Hubble time.) Put $\theta=-H$. A new dimensionless variable $\tau$ (not related to the $\tau$ of Proposition 3.15) is defined by

$$
\frac{d t}{d \tau}=\frac{3}{\theta}
$$

We normalize so that $\tau=0$ corresponds to $t=t_{0}$. Then

$$
g=-\left(\frac{3}{\theta}\right)^{2} d \tau^{2}+h(\tau)
$$

That is, the lapse function is

$$
L(\tau)=\frac{3}{\theta} .
$$


Approaching the singularity corresponds to $\tau \rightarrow-\infty$.

As the spacetime Ricci tensor vanishes, the curvature tensor is determined by the spacetime Weyl curvature. Since $\operatorname{dim}(X)=3$, the Weyl curvature is expressed in terms of "electric" and "magnetic" tensors [11, Section 1.1.3]. After normalization by the Hubble time, the tensor components can be written as polynomials in the Wainwright-Hsu variables $\Sigma_{+}, \Sigma_{-}, N_{+}, N_{-}, N_{1}$ [11, (6.37)]. Hence the Einstein flow will be type-I provided that these variables remain bounded as one approaches the singularity. From [24], this is the case.

The normalized spatial scalar curvature is

$$
\frac{R}{H^{2}}=-\frac{1}{2}\left[N_{1}^{2}+N_{2}^{2}+N_{3}^{2}-2\left(N_{1} N_{2}+N_{2} N_{3}+N_{3} N_{1}\right)\right]
$$

Going toward the singularity, the flow approaches an attractor where two of the $N_{i}$ 's vanish [24]. Hence the Einstein flow has asymptotically nonpositive spatial scalar curvature.

We now restrict to a certain class of Mixmaster flows. The Kasner circle is $\left\{\left(\Sigma_{+}, \Sigma_{-}\right)\right.$: $\left.\Sigma_{+}^{2}+\Sigma_{-}^{2}=1\right\}$. The Kasner map is a certain degree two map of the Kasner circle to itself [11, Section 6.4.1]. Given a periodic orbit of the Kasner map that is not a fixed point, there is a heteroclinic cycle of the ODE that consists of the periodic points on the Kasner circle, joined by Taub type-II Einstein flows that asymptotically approach two adjacent points in the orbit as time goes to $\pm \infty$. There is a family of Mixmaster flows that asymptotically approach the heteroclinic cycle as $\tau \rightarrow-\infty$ [17].

Proposition 4.23. These Mixmaster flows satisfy the assumptions of Proposition 3.15.

Proof. We first show that $\operatorname{dvol}_{h\left(t_{H}\right)}(x)$ fails to be $O\left(t_{H}^{1+\beta}\right)$ for any $\beta>0$, where the $t_{H}$ denotes the Hubble time. The variable $\tau$ is defined in a way that there is a simple dependence of the volume form on $\tau$. Namely,

$$
\operatorname{dvol}_{h(\tau)}(x)=e^{3 \tau} \operatorname{dvol}_{h(0)}(x) .
$$

To see this, equation (2.4) gives

$$
\frac{d}{d \tau} \operatorname{dvol}_{h(\tau)}(x)=-L H \operatorname{dvol}_{h(\tau)}(x) .
$$

Then (4.21) implies

$$
\frac{d}{d \tau} \operatorname{dvol}_{h(\tau)}(x)=3 \operatorname{dvol}_{h(\tau)}(x)
$$

from which (4.24) follows.

Using (4.24), to see how $\operatorname{dvol}_{h(\tau)}(x)$ depends on the Hubble time $t_{H}$, since $t_{H}=\frac{3}{\theta}$ it suffices to see how $\theta$ depends on $\tau$. One has

$$
\frac{d \theta}{d \tau}=-\left(1+2 \Sigma_{+}^{2}+2 \Sigma_{-}^{2}\right) \theta
$$

So

$$
\log \theta(\tau)-\log \theta(0)=-3 \tau+2 \int_{\tau}^{0}\left(\Sigma_{+}^{2}+\Sigma_{-}^{2}-1\right)(u) d u
$$


From [17, Sections 3 and 4], as $\tau \rightarrow-\infty$, the trajectory in the $\left(\Sigma_{+}, \Sigma_{-}\right)$-plane will spend almost all of its time near the periodic orbit on the Kasner circle. Consequently, $\int_{\tau}^{0}\left(\Sigma_{+}^{2}+\Sigma_{-}^{2}-1\right)(u) d u$ will be sublinear in $|\tau|$ as $\tau \rightarrow-\infty$. Thus to leading order, $\theta(\tau)$ will go like $e^{-3 \tau}$. Then for any $\beta>0$, it follows that $\operatorname{dvol}_{h(\tau)}(x)$ will fail to be $O\left(t_{H}^{1+\beta}\right)$ as $\tau \rightarrow-\infty$, i.e. as $t_{H} \rightarrow 0$.

We now show that the Einstein flow is noncollapsing at $x$ as $t_{H} \rightarrow 0$. Because the Einstein flow is type-I, the rescaling $t_{H}^{-2} h\left(t_{H}\right)$ of the metric at Hubble time $t_{H}$ has a double sided curvature bound that is independent of $t_{H}$. Suppose that the Einstein flow is collapsing at $x$, as witnessed by a sequence of times $\left\{t_{H}^{j}\right\}_{j=1}^{\infty}$ going to zero. From the theory of bounded curvature collapse [8], there is some constant $a>0$ so that for large $j$, there is a loop at $x$ that is homotopically nontrivial in the metric ball $B_{\left(t_{H}^{j}\right)^{-2} h\left(t_{H}^{j}\right)}(x, a)$, with the length of the loop (with respect to $\left(t_{H}^{j}\right)^{-2} h\left(t_{H}^{j}\right)$ ) going to zero as $j \rightarrow \infty$.

On the other hand, the evolution of $h$ is given by $(2.4)$. Let $\left\{\sigma^{i}\right\}_{i=1}^{3}$ be the coframe to the orthonormal frame $\left\{e_{i}\right\}_{i=1}^{3}$ used in deriving the ODE, so

$$
h=\sigma^{1} \otimes \sigma^{1}+\sigma^{2} \otimes \sigma^{2}+\sigma^{3} \otimes \sigma^{3} .
$$

Then

$$
\begin{aligned}
K= & \frac{H}{3}\left(1-2 \Sigma_{+}\right) \sigma^{1} \otimes \sigma^{1}+H\left(\frac{1}{3}+\frac{1}{3} \Sigma_{+}+\frac{1}{\sqrt{3}} \Sigma_{-}\right) \sigma^{2} \otimes \sigma^{2}+ \\
& H\left(\frac{1}{3}+\frac{1}{3} \Sigma_{+}-\frac{1}{\sqrt{3}} \Sigma_{-}\right) \sigma^{3} \otimes \sigma^{3} .
\end{aligned}
$$

Using (2.4) and (4.27), one obtains

$$
\begin{aligned}
\frac{d\left(H^{2} h\right)}{d \tau}= & \left(-4 \Sigma_{+}-4 \Sigma_{+}^{2}-4 \Sigma_{-}^{2}\right) H^{2} \sigma^{1} \otimes \sigma^{1}+ \\
& \left(2 \Sigma_{+}+2 \sqrt{3} \Sigma_{-}-4 \Sigma_{+}^{2}-4 \Sigma_{-}^{2}\right) H^{2} \sigma^{2} \otimes \sigma^{2}+ \\
& \left(2 \Sigma_{+}-2 \sqrt{3} \Sigma_{-}-4 \Sigma_{+}^{2}-4 \Sigma_{-}^{2}\right) H^{2} \sigma^{2} \otimes \sigma^{2} .
\end{aligned}
$$

The region in the $\left(\Sigma_{+}, \Sigma_{-}\right)$-plane where $-4 \Sigma_{+}-4 \Sigma_{+}^{2}-4 \Sigma_{-}^{2} \geq 0$ is the closed disk with center $\left(-\frac{1}{2}, 0\right)$ and radius $\frac{1}{2}$. The regions where the other coefficients are nonnegative are the rotations of this disk around the origin by $\frac{2 \pi}{3}$ and $\frac{4 \pi}{3}$ radians. In particular, these three disks only meet the Kasner circle at the Taub points $\left\{e^{\frac{i \pi}{3}}, e^{i \pi}, e^{\frac{5 \pi}{3}}\right\}$, which correspond to flat Kasner spacetimes. Since the heteroclinic cycle avoids these points, as $\tau \rightarrow-\infty$ the normalized metric $\theta^{2} h$ will be greatly expanded during the time spent near the Kasner circle and will have bounded contraction the rest of the time, again using [17, Sections 3 and 4]. Hence there is a large overall expansion and so at the fixed time $\tau=0$, there is a sequence of loops at $x$ that are homotopically nontrivial and whose lengths go to zero. This is a contradiction.

Because of the homogeneity, in this case the Kasner-like regions in the conclusion of Proposition 3.15 are standard Kasner geometries in the sense of Example 4.5. It seems 
plausible that the hypotheses of Proposition 3.15 are also satisfied for the Mixmaster spacetimes considered in [4, 7].

Example 4.32. Consider a locally homogeneous Einstein flow of Bianchi type VIII. The spatial geometry is a quotient of $\widehat{\mathrm{SL}(2, \mathbb{R})}$. The spatial scalar curvature is nonpositive.

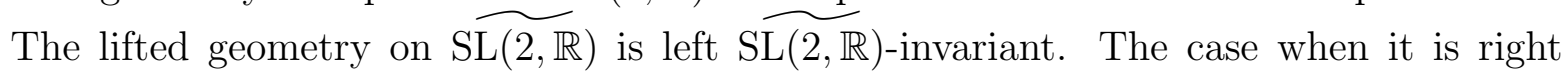

$\widehat{\mathrm{SO}(2)}$-invariant was essentially considered in Example 4.11, so we assume that the Einstein flow is not right $\widetilde{\mathrm{SO}(2)}$-invariant.

For a generic set of initial conditions, a Bianchi VIII solution converges as $\tau \rightarrow-\infty$ to the Mixmaster attractor [7]. Then Proposition 4.17] extends to these solutions. As mentioned in [17], the results of that paper extend to the construction of Bianchi VIII solutions that, as $\tau \rightarrow-\infty$, approach a heteroclinic cycle coming from a periodic orbit of the Kasner map. (These solutions are in the generic set of [7].) Then Proposition 4.23 extends to such solutions.

\section{REFERENCES}

[1] M. Anderson, "On long-time evolution in general relativity and geometrization of 3-manifolds", Comm. Math. Phys. 222, p. 533-567 (2001)

[2] L. Andersson and V. Moncrief, "Elliptic-hyperbolic systems and the Einstein equations", Ann. Henri Poincaré 4, p. 1-34 (2003)

[3] L. Andersson and V. Moncrief, "Einstein spaces as attractors for the Einstein flow", J. Diff. Geom. 89, p. 1-47 (2011)

[4] F. Béguin, "Aperiodic oscillatory asymptotic behavior for some Bianchi spacetimes", Class. and Quantum Gravity 27, 185005 (2010)

[5] V. Belinskii, I, Khalatnikov and E. Lifshitz, "Oscillatory approach to a singular point in the relativistic cosmology", Adv. Phys. 19, p. 525-573 (1970)

[6] V. Belinski and M. Henneaux, The cosmological singularity, Cambridge University Press, Cambridge (2017)

[7] B. Brehm, "Bianchi VIII and IX vacuum cosmologies: Almost every solution forms particle horizons and converges to the Mixmaster attractor", preprint, https://arxiv.org/abs/1606.08058 (2016)

[8] J. Cheeger, K. Fukaya and M. Gromov, "Nilpotent structures and invariant metrics on collapsed manifolds" J. Amer. Math. Soc. 5, 327372 (1992)

[9] T. Dutilleul, "Chaotic dynamics of spatially homogeneous spacetimes", https://tel.archivesouvertes.fr/tel-02488655/document (2019)

[10] D. Eardley and L. Smarr, "Time functions in numerical relativity: Marginally bound dust collapse", Phys. Rev. D 19, p. 2239-2259 (1979)

[11] G. Ellis and J. Wainwright, Dynamical systems in cosmology, Cambridge University Press, Cambridge (1997)

[12] A. Fischer and V. Moncrief, "Hamiltonian reduction and perturbations of continuously self-similar $(n+1)$-dimensional Einstein vacuum spacetimes", Class. Quantum Grav. 19, p. 5557-5589 (2002)

[13] C. Gerhardt, " $H$-surfaces in Lorentzian manifolds", Comm. Math. Phys. 89, p. 523-553 (1983)

[14] S. Hawking and G. Ellis, The Large Scale Structure of Space-Time, Cambridge University Press, Cambridge (1973)

[15] J. Isenberg, "On strong cosmic censorship", in Surveys in Differential Geometry XX, International Press of Boston, p. 17-36 (2015) 
[16] J. Isenberg and V. Moncrief, "Asymptotic behavior of the gravitational field and the nature of singularities in Gowdy spacetimes", Adv. Phys. 199, p. 84-122 (1990)

[17] S. Liebscher, J. Härterich, K. Webster and M. Georgi, "Ancient dynamics in Bianchi models: Approach to periodic cycles", Comm. Math. Phys. 305, p. 59-83 (2011)

[18] J. Lott, "On the long-time behavior of type-III Ricci flow solutions", Math. Ann. 339, p. 627 (2007)

[19] J. Lott, "Collapsing in the Einstein flow", Annales Henri Poincare 19, p. 2245-2296 (2018)

[20] J. Lott, "On the initial geometry of a vacuum cosmological spacetime", Class. and Quantum Gravity $37,085017(2020)$

[21] J. Marsden and F. Tipler, "Maximal hypersurfaces and foliations of mean curvature in general relativity", Phys. Rep. 66, p. 109-139 (1980)

[22] C. Misner, K. Thorne and J. Wheeler, Gravitation, W. H. Freeman, San Francisco (1973)

[23] P. Petersen, Riemannian Geometry, $3^{r d}$ edition, Springer, New York (2016)

[24] H. Ringström, "The Bianchi IX attractor", Annales Henri Poincaré 2, p. 405-500 (2001)

[25] P. Scott, "The geometries of 3-manifolds", Bull. London Math. Soc. 15, p. 401-487 (1983)

Department of Mathematics, University of California, Berkeley, Berkeley, CA 947203840 , USA

Email address: lott@berkeley.edu 